\title{
Activity of the novel siderophore cephalosporin cefiderocol against multidrug-resistant Gram-negative pathogens
}

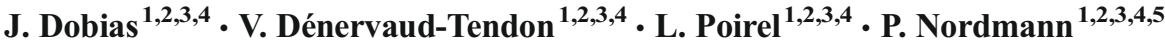

\begin{abstract}
The novel siderophore cephalosporin cefiderocol (S-649266) with potent activity against Gram-negative pathogens was recently developed (Shionogi \& Co., Ltd.). Here, we evaluated the activity of this new molecule and comparators against a collection of previously characterized Gram-negative isolates using broth microdilution panels. A total of 753 clinical multidrug-resistant Gram-negative isolates collected from hospitals worldwide were tested against cefiderocol and antibiotic comparators (ceftolozane-tazobactam [CT], meropenem [MEM], ceftazidime [CAZ], ceftazidime-avibactam [CZA], colistin [CST], aztreonam [ATM], amikacin [AMK], ciprofloxacin [CIP], cefepime [FEP], and tigecycline [TGC]) for their susceptibility. The collection included Escherichia coli ( $n=164)$, Klebsiella pneumoniae $(n=298)$, Enterobacter $\mathrm{sp}$. ( $n=159)$, Pseudomonas aeruginosa $(n=45)$, and Acinetobacter baumannii $(n=87)$. Resistance mechanisms included producers of carbapenemases and extended-spectrum $\beta$-lactamases (ESBLs). In addition, a series of colistinresistant enterobacterial isolates $(n=74)$, including 15 MCR1 producers, were tested. The $\mathrm{MIC}_{90}$ of cefiderocol was $2 \mathrm{mg} /$
\end{abstract}

P. Nordmann

patrice.nordmann@unifr.ch

1 Emerging Antibiotic Resistance Unit, University of Fribourg, Fribourg, Switzerland

2 National Reference Center for Emerging Antibiotic Resistance (Switzerland), University of Fribourg, Fribourg, Switzerland

3 French INSERM European Unit (LEA/IAME), University of Fribourg, Fribourg, Switzerland

4 Medical and Molecular Microbiology Unit, Department of Medicine, University of Fribourg, rue Albert Gockel 3, 1700 Fribourg, Switzerland

5 Institute for Microbiology, University of Lausanne and University Hospital Centre, Lausanne, Switzerland
$\mathrm{L}$, while those of comparative drugs were $>64 \mathrm{mg} / \mathrm{L}$ for $\mathrm{CT}$, MEM, CAZ, CZA, and AMK, $>32 \mathrm{mg} / \mathrm{L}$ for ATM, $>16 \mathrm{mg} / \mathrm{L}$ for FEP, $8 \mathrm{mg} / \mathrm{L}$ for CST, and $2 \mathrm{mg} / \mathrm{L}$ for TGC. The $\mathrm{MIC}_{50}$ of cefiderocol was $0.5 \mathrm{mg} / \mathrm{L}$, while those of other drugs were $>64 \mathrm{mg} / \mathrm{L}$ for CAZ, $64 \mathrm{mg} / \mathrm{L}$ for CT, >32 mg/L for ATM, $>16 \mathrm{mg} / \mathrm{L}$ for FEP, $8 \mathrm{mg} / \mathrm{L}$ for MEM and AMK, $>4 \mathrm{mg} / \mathrm{L}$ for CIP, $1 \mathrm{mg} / \mathrm{L}$ for CZA, $0.5 \mathrm{mg} / \mathrm{L}$ for TGC, and $<0.5 \mathrm{mg} / \mathrm{L}$ for CST. Only 20 out of 753 strains showed MIC values of cefiderocol $\geq 8 \mu \mathrm{g} / \mathrm{mL}$. Compared to the other drugs tested, cefiderocol was more active, with the exception of colistin and tigecycline showing equivalent activity against certain subgroups of bacteria.

\section{Introduction}

Gram-negative bacteria that produce extended-spectrum $\beta$ lactamases (ESBLs) are a major concern in healthcare due to their ability to spread globally [1]. ESBLs are a major group of enzymes that confer resistance to several generations of $\beta$ lactam antibiotics, including third-generation cephalosporins $[2,3]$. ESBL-encoding genes that are primarily plasmidencoded include mostly TEM-, SHV-, and CTX-M-type enzymes [4]. Enterobacteriaceae, such as Klebsiella pneumoniae and Escherichia coli, are the main ESBL producers that have been reported globally. Carbapenems are mostly used for the treatment of infections due to ESBL-producing bacteria, but carbapenemase-producing bacteria are now extensively reported $[5,6]$. Polymyxins (e.g., colistin and polymyxin B) are now more frequently used as last-resort antibiotics for treating patients with multidrug-resistant bacterial infections [7]. However, a recent report shows that a novel gene ( $m c r-1)$ as a source of plasmid-encoded resistance encoded on a plasmid in E. coli may quickly spread to other bacterial strains or species [8-10]. Therefore, due to the increasing threat of 
multidrug-resistant and pandrug-resistant bacteria, there is a need for novel molecules. Cefiderocol (CFDC) is a novel parenteral siderophore cephalosporin also known as S-649266. It possesses a unique mechanism for penetrating efficiently into Gram-negative pathogens. It uses a "Trojan horse" strategy by binding free iron and is then actively transported into bacterial cells across the outer membrane of Gram-negative bacteria by way of the iron-transport system [11, 12]. Cefiderocol is a cephalosporin molecule with an attached catechol moiety on the 3-position side chain which binds to ferric iron [11]. Once across the outer membrane, the iron dissociates and the cephalosporin binds to penicillin-binding proteins (PBP), mainly PBP3, as other cephalosporins do to disrupt cell wall synthesis $[11,13]$, contributing to a potent antimicrobial activity against Gram-negative bacteria. In addition, this antimicrobial activity of cefiderocol is enhanced by the high stability of cefiderocol to hydrolysis by nearly all $\beta$-lactamases, including both the serine and metallo-carbapenemases [14]. The ability to cross the outer membrane through the active iron-transport system overcomes resistance due to porin channel mutations and efflux pump overproducers. It results that cefiderocol exhibits potent in vitro and in vivo activity against all species of Gramnegative bacteria, including carbapenem-resistant strains of Enterobacteriaceae, Pseudomonas aeruginosa, and Acinetobacter baumannii, and even Stenotrophomonas maltophilia [15]. Here, we evaluated the antimicrobial activity of cefiderocol and other Gram-negative antibiotics (aztreonam, amikacin, cefepime, ceftazidime, ceftazidime-avibactam, ceftolozane-tazobactam, ciprofloxacin, meropenem, colistin, and tigecycline) against a panel of 753 multidrug-resistant bacterial isolates from human clinical sources with characterized antibiotic resistance mechanisms.

\section{Materials and methods}

\section{Bacterial isolates}

A total of 753 clinical multidrug-resistant isolates were evaluated in this study (Table 1). They were representative of the most widespread and broad-spectrum mechanisms of resistance currently observed worldwide in Gram-negative bacteria. The strains were collected from hospitals worldwide (42 countries) from 2000 to 2016 , with a majority dating from the 2012-2016 period. They were of various origins (not always recorded) but mostly from urines, broncho-alveolar specimens, blood, pus, and stools.

\section{In vitro susceptibility test methods}

Minimum inhibitory concentrations (MICs) were determined following the Clinical and Laboratory Standards Institute (CLSI) broth microdilution (BMD) guidelines [16]. Frozen
Table 1 Bacterial strains tested in this study

\begin{tabular}{|c|c|c|}
\hline Genus (species) & $\begin{array}{l}\text { Number of } \\
\text { tested isolates }\end{array}$ & Characterized resistance \\
\hline \multicolumn{3}{|c|}{ Escherichia coli (164) } \\
\hline & 43 & OXA $(-1 / 48 / 181 / 204)$ \\
\hline & 22 & VIM (-1/2/4/19), IMP (-1/8) \\
\hline & 45 & NDM (-1/4/5/6/7) \\
\hline & 11 & $\mathrm{KPC}(-2 / 3)$ \\
\hline & 25 & CTX-M (-1/3/15) \\
\hline & 15 & MCR-1 \\
\hline & 3 & Non-MCR-1 colistin resistant \\
\hline \multicolumn{3}{|c|}{ Klebsiella pneumoniae (298) } \\
\hline & 101 & $\mathrm{KPC}(-2 / 3 / 11)$ \\
\hline & 89 & OXA $(-48 / 162 / 163 / 181 / 204 / 232)^{a}$ \\
\hline & 18 & $\operatorname{NDM}^{\mathrm{b}}(-1 / 4)$ \\
\hline & 20 & VIM (-1/4/19), IMP (-1/4/8) \\
\hline & 25 & CTX-M (-3/15) \\
\hline & 45 & Colistin R \\
\hline \multicolumn{3}{|c|}{ Enterobacteriaceae (159) } \\
\hline & 26 & OXA $(-48 / 163)$ \\
\hline & 10 & NDM-1 \\
\hline & 5 & VIM-1 \\
\hline & 14 & IMP (-1/8) \\
\hline & 14 & $\mathrm{KPC}-2$ \\
\hline & 49 & CTX-M (-3/15) \\
\hline & 7 & VEB-1 \\
\hline & 14 & SHV-12 \\
\hline & 9 & $\begin{array}{c}\text { Plasmid-mediated AmpC and } \\
\text { overproducer of AmpC }\end{array}$ \\
\hline & 11 & Colistin R \\
\hline \multicolumn{3}{|c|}{ Pseudomonas aeruginosa (45) } \\
\hline & 6 & PER-1 \\
\hline & 9 & SHV (-2a/5/12), GES (-1/9) \\
\hline & 20 & $\operatorname{IMP}(-1 / 2 / 10 / 13 / 15 / 19 / 29), \mathrm{KPC}-2$ \\
\hline & 10 & VIM (-1/2), SPM-1, GIM-1 \\
\hline \multicolumn{3}{|c|}{ Acinetobacter baumannii (87) } \\
\hline & 85 & OXA-23/40/58/72 \\
\hline & 2 & NDM-1, IMP-4 \\
\hline Total & 753 & \\
\hline
\end{tabular}

${ }^{\text {a }}$ Five strains are OXA-48 producers and colistin resistant

${ }^{\mathrm{b}}$ Five strains are NDM producers and colistin resistant

96-well broth microdilution panels with pre-loaded antibiotic-growth medium were supplied by International Health Management Associates, Inc. (Schaumburg, IL, USA). Cefiderocol was tested in iron-depleted cation-adjusted Mueller-Hinton broth (ID-CAMHB) [17], as recently approved by the CLSI (http://clsi.org/standards/micro/ microbiology-files/), whereas comparators were tested in cation-adjusted Mueller-Hinton broth (CAMHB). Quality 
Table 2 In vitro activities of cefiderocol (CFDC), ceftolozanetazobactam (CT), meropenem (MEM), ceftazidime (CAZ), ceftazidime-avibactam (CZA), colistin (CST), aztreonam (ATM), amikacin (AMK), ciprofloxacin (CIP), cefepime (FEP), and tigecycline (TGC) against $E$. coli, $K$. pneumoniae, Enterobacter sp., $P$. aeruginosa, and $A$. baumannii

\begin{tabular}{|c|c|c|c|c|}
\hline $\begin{array}{l}\text { Organism group/ } \\
\text { antimicrobial agent }\end{array}$ & MIC range $(\mathrm{mg} / \mathrm{L})$ & No. & $\mathrm{MIC}_{50}{ }^{\mathrm{a}}$ & $\mathrm{MIC}_{90}{ }^{\mathrm{a}}$ \\
\hline \multicolumn{5}{|l|}{ All species } \\
\hline CFDC & $0.03-64$ & 753 & 0.5 & 2 \\
\hline CT & $0.03-64$ & 753 & 64 & $>64$ \\
\hline CAZ & $0.03-64$ & 753 & $>64$ & $>64$ \\
\hline CZA & $0.03-64$ & 753 & 1 & $>64$ \\
\hline FEP & $0.05-16$ & 753 & $>16$ & $>16$ \\
\hline MEM & $0.03-64$ & 753 & 8 & $>64$ \\
\hline CIP & $0.25-4$ & 753 & $>4$ & $>4$ \\
\hline ATM & $0.05-32$ & 753 & $>32$ & $>32$ \\
\hline AMK & $4-64$ & 753 & 8 & $>64$ \\
\hline CST & $0.5-8$ & 753 & $\leq 0.5$ & 8 \\
\hline TGC & $0.25-4$ & 753 & 0.5 & 2 \\
\hline \multicolumn{5}{|l|}{ Escherichia coli } \\
\hline CFDC & $0.03-64$ & 164 & 0.5 & 4 \\
\hline $\mathrm{CT}$ & $0.03-64$ & 164 & 8 & $>64$ \\
\hline CAZ & $0.03-64$ & 164 & 64 & $>64$ \\
\hline $\mathrm{CZA}$ & $0.03-64$ & 164 & 0.5 & $>64$ \\
\hline FEP & $0.05-16$ & 164 & $>16$ & $>16$ \\
\hline MEM & $0.03-64$ & 164 & 2 & $>64$ \\
\hline CIP & $0.25-4$ & 164 & $>4$ & $>4$ \\
\hline ATM & $0.05-32$ & 164 & $>32$ & $>32$ \\
\hline AMK & $4-64$ & 164 & $\leq 4$ & $>64$ \\
\hline CST & $0.5-8$ & 164 & $\leq 0.5$ & 2 \\
\hline TGC & $0.25-4$ & 164 & $\leq 0.25$ & $\leq 0.25$ \\
\hline \multicolumn{5}{|c|}{ Klebsiella pneumoniae } \\
\hline CFDC & $0.03-64$ & 298 & 1 & 2 \\
\hline $\mathrm{CT}$ & $0.03-64$ & 298 & 64 & $>64$ \\
\hline CAZ & $0.03-64$ & 298 & $>64$ & $>64$ \\
\hline $\mathrm{CZA}$ & $0.03-64$ & 298 & 1 & $>64$ \\
\hline FEP & $0.05-16$ & 298 & $>16$ & $>16$ \\
\hline MEM & $0.03-64$ & 298 & 16 & $>64$ \\
\hline CIP & $0.25-4$ & 298 & $>4$ & $>4$ \\
\hline ATM & $0.05-32$ & 298 & $>32$ & $>32$ \\
\hline AMK & $4-64$ & 298 & 8 & 64 \\
\hline CST & $0.5-8$ & 298 & $\leq 0.5$ & 16 \\
\hline TGC & $0.25-4$ & 298 & 0.5 & 1 \\
\hline \multicolumn{5}{|l|}{ Enterobacter sp. } \\
\hline CFDC & $0.03-64$ & 159 & 0.5 & 4 \\
\hline $\mathrm{CT}$ & $0.03-64$ & 159 & 16 & $>64$ \\
\hline $\mathrm{CAZ}$ & $0.03-64$ & 159 & $>64$ & $>64$ \\
\hline $\mathrm{CZA}$ & $0.03-64$ & 159 & 0.5 & $>64$ \\
\hline FEP & $0.05-16$ & 159 & $>16$ & $>16$ \\
\hline MEM & $0.03-64$ & 159 & 0.5 & 16 \\
\hline CIP & $0.25-4$ & 159 & $>4$ & $>4$ \\
\hline ATM & $0.05-32$ & 159 & $>32$ & $>32$ \\
\hline AMK & $4-64$ & 159 & $\leq 4$ & 16 \\
\hline CST & $0.5-8$ & 159 & $\leq 0.5$ & 8 \\
\hline TGC & $0.25-4$ & 159 & 0.5 & 1 \\
\hline
\end{tabular}


Table 2 (continued)

\begin{tabular}{|c|c|c|c|c|}
\hline $\begin{array}{l}\text { Organism group/ } \\
\text { antimicrobial agent }\end{array}$ & MIC range $(\mathrm{mg} / \mathrm{L})$ & No. & $\mathrm{MIC}_{50}{ }^{\mathrm{a}}$ & $\mathrm{MIC}_{90}{ }^{2}$ \\
\hline \multicolumn{5}{|c|}{ Pseudomonas aeruginosa } \\
\hline CFDC & $0.03-64$ & 45 & 0.5 & 2 \\
\hline $\mathrm{CT}$ & $0.03-64$ & 45 & 64 & $>64$ \\
\hline CAZ & $0.03-64$ & 45 & $>64$ & $>64$ \\
\hline $\mathrm{CZA}$ & $0.03-64$ & 45 & 16 & $>64$ \\
\hline FEP & $0.05-16$ & 45 & $>16$ & $>16$ \\
\hline MEM & $0.03-64$ & 45 & 32 & $>64$ \\
\hline CIP & $0.25-4$ & 45 & $>4$ & $>4$ \\
\hline ATM & $0.05-32$ & 45 & 16 & $>32$ \\
\hline AMK & $4-64$ & 45 & 8 & $>64$ \\
\hline CST & $0.5-8$ & 45 & $\leq 0.5$ & 1 \\
\hline TGC & $0.25-4$ & 45 & $>4$ & $>4$ \\
\hline \multicolumn{5}{|c|}{ Acinetobacter baumannii } \\
\hline CFDC & $0.03-64$ & 87 & 0.12 & 4 \\
\hline $\mathrm{CT}$ & $0.03-64$ & 87 & 16 & $>64$ \\
\hline CAZ & $0.03-64$ & 87 & $>64$ & $>64$ \\
\hline $\mathrm{CZA}$ & $0.03-64$ & 87 & 16 & 64 \\
\hline FEP & $0.05-16$ & 87 & $>16$ & $>16$ \\
\hline MEM & $0.03-64$ & 87 & 32 & 64 \\
\hline CIP & $0.25-4$ & 87 & $>4$ & $>4$ \\
\hline ATM & $0.05-32$ & 87 & $>32$ & $>32$ \\
\hline AMK & 4-64 & 87 & 64 & $>64$ \\
\hline CST & $0.5-8$ & 87 & $\leq 0.5$ & 1 \\
\hline TGC & $0.25-4$ & 87 & 1 & 2 \\
\hline
\end{tabular}

${ }^{a} \mathrm{MIC}$, minimum inhibitory concentration; $\mathrm{MIC}_{50 / 90}$, MIC that inhibits $50 \%$ and $90 \%$ of the isolates, respectively

control (QC) strains (E. coli ATCC 25922, K. pneumoniae ATCC 700603, and P. aeruginosa ATCC 27853) were tested regularly to ensure the stability of the panels and validity of the test methods. The acceptable concentration range for QC strains is $0.06-0.5 \mathrm{mg} / \mathrm{L}$ for $E$. coli and $P$. aeruginosa and not yet defined for $K$. pneumoniae.

All isolates were tested against the following antibiotics for the given concentration range: cefiderocol (S-649266) (CFDC, 0.03-64 mg/L), aztreonam (ATM, 0.5-32 mg/L), cefepime (FEP, 0.5-16 mg/L), ceftolozane-tazobactam (CT, 0.03-64 mg/L), meropenem (MEM, 0.03-64 mg/L), ceftazidime (CAZ, 0.03-64 mg/L), ceftazidime-avibactam (CZA, 0.03-64 mg/L), colistin (CST, 0.5-8 mg/L), amikacin (AMK, 4-64 mg/L), ciprofloxacin (CIP, 0.25-4 mg/L), and tigecycline (TGC, $0.25-4 \mathrm{mg} / \mathrm{L}$ ).

The strains were grown overnight on a non-selective agar medium and two to three colonies were solubilized in $3 \mathrm{~mL}$ sterile $0.85 \% \mathrm{NaCl}$. The turbidity was adjusted to 0.5 McFarland with a McFarland densitometer DEN-1B from Grant Bio (Grant instruments Ltd., Cambridge, UK). One milliliter of inoculum was then diluted in $29 \mathrm{~mL}$ of sterile $\mathrm{ddH}_{2} \mathrm{O}$ and $10 \mu \mathrm{L}$ were added to each BMD panel well. The panels were incubated for $16-20 \mathrm{~h}$ at $35^{\circ} \mathrm{C}$. To ensure an even thermal distribution during incubation, the panels were stacked no more than four high. The MIC reading was then done according to the CLSI guidelines [18].

\section{Results and discussion}

The susceptibility test results are listed in Tables 2-7. The $\mathrm{MIC}_{90}$ (MIC value that inhibits $90 \%$ of the isolates) of cefiderocol was $2 \mathrm{mg} / \mathrm{L}$ (Table 2), while those of comparative drugs were $>64 \mathrm{mg} / \mathrm{L}$ for CT, MEM, CAZ, CZA, and AMK, $>32 \mathrm{mg} / \mathrm{L}$ for ATM, >16 mg/L for FEP, $8 \mathrm{mg} / \mathrm{L}$ for CST, and $2 \mathrm{mg} / \mathrm{L}$ for TGC. The $\mathrm{MIC}_{50}$ of CFDC was at $0.5 \mathrm{mg} / \mathrm{L}$, while those of other drugs were $>64 \mathrm{mg} / \mathrm{L}$ for CAZ, $64 \mathrm{mg} / \mathrm{L}$ for CT, $>32 \mathrm{mg} / \mathrm{L}$ for ATM, > $16 \mathrm{mg} / \mathrm{L}$ for FEP, $8 \mathrm{mg} / \mathrm{L}$ for MEM and AMK, $>4 \mathrm{mg} / \mathrm{L}$ for CIP, $1 \mathrm{mg} / \mathrm{L}$ for CZA, $0.5 \mathrm{mg} / \mathrm{L}$ for TGC, and $\leq 0.5 \mathrm{mg} / \mathrm{L}$ for CST. The addition of $4 \mu \mathrm{g} / \mathrm{mL}$ of avibactam restored the activities of ceftazidime against the majority of the tested enterobacterial isolates.

The $\mathrm{MIC}_{50}$ and $\mathrm{MIC}_{90}$ of CFDC for Enterobacteriaceae producing KPC carbapenemases were $\leq 1 \mu \mathrm{g} / \mathrm{mL}$ and $\leq 2 \mathrm{mg}$ / $\mathrm{L}$, respectively (Table 3 ). The only competitive comparators were ceftazidime-avibactam $\left(\mathrm{MIC}_{50 / 90}\right)(1 / 4)$, colistin $(\leq 0.5 /$ 
Table 3 In vitro activities of cefiderocol (CFDC), ceftolozanetazobactam (CT), ceftazidime (CAZ), ceftazidime-avibactam (CZA), cefepime (FEP), meropenem (MEM) ciprofloxacin (CIP), aztreonam (ATM), amikacin (AMK), colistin (CST), and tigecycline (TGC) against Enterobacteriaceae

(E. coli, K. pneumoniae, and Enterobacter sp.) producing a KPC-type carbapenemase

\begin{tabular}{|c|c|c|c|c|}
\hline Organism group/antimicrobial agent & MIC range $(\mathrm{mg} / \mathrm{L})$ & No. & $\mathrm{MIC}_{50}{ }^{\mathrm{a}}$ & $\mathrm{MIC}_{90}{ }^{\mathrm{a}}$ \\
\hline \multicolumn{5}{|l|}{ Total Enterobacteriaceae } \\
\hline CFDC & $0.03-64$ & 127 & 1 & 2 \\
\hline $\mathrm{CT}$ & $0.03-64$ & 127 & 64 & $>64$ \\
\hline CAZ & $0.03-64$ & 127 & $>64$ & $>64$ \\
\hline CZA & $0.03-64$ & 127 & 1 & 4 \\
\hline FEP & $0.05-16$ & 127 & $>16$ & $>16$ \\
\hline MEM & $0.03-64$ & 127 & 32 & $>64$ \\
\hline CIP & $0.25-4$ & 127 & $>4$ & $>4$ \\
\hline ATM & $0.05-32$ & 127 & $>32$ & $>32$ \\
\hline AMK & $4-64$ & 127 & 16 & 32 \\
\hline CST & $0.5-8$ & 127 & $\leq 0.5$ & $>8$ \\
\hline TGC & $0.25-4$ & 127 & 0.5 & 1 \\
\hline \multicolumn{5}{|l|}{ Escherichia coli } \\
\hline CFDC & $0.03-64$ & 12 & 0.5 & 1 \\
\hline $\mathrm{CT}$ & $0.03-64$ & 12 & 32 & $>64$ \\
\hline $\mathrm{CAZ}$ & $0.03-64$ & 12 & 64 & $>64$ \\
\hline $\mathrm{CZA}$ & $0.03-64$ & 12 & 1 & 4 \\
\hline FEP & $0.05-16$ & 12 & $>16$ & $>16$ \\
\hline MEM & $0.03-64$ & 12 & 8 & 64 \\
\hline CIP & $0.25-4$ & 12 & $>4$ & $>4$ \\
\hline ATM & $0.05-32$ & 12 & 64 & 64 \\
\hline AMK & $4-64$ & 12 & $\leq 4$ & $>64$ \\
\hline $\mathrm{CST}$ & $0.5-8$ & 12 & $\leq 0.5$ & $\leq 0.5$ \\
\hline TGC & $0.25-4$ & 12 & $\leq 0.25$ & $\leq 0.25$ \\
\hline \multicolumn{5}{|l|}{ Klebsiella pneumoniae } \\
\hline CFDC & $0.03-64$ & 101 & 1 & 2 \\
\hline $\mathrm{CT}$ & $0.03-64$ & 101 & 64 & $>64$ \\
\hline CAZ & $0.03-64$ & 101 & $>64$ & $>64$ \\
\hline CZA & $0.03-64$ & 101 & 2 & 4 \\
\hline FEP & $0.05-16$ & 101 & $>16$ & $>16$ \\
\hline MEM & $0.03-64$ & 101 & 64 & $>64$ \\
\hline CIP & $0.25-4$ & 101 & $>4$ & $>4$ \\
\hline ATM & $0.05-32$ & 101 & $>32$ & $>32$ \\
\hline AMK & $4-64$ & 101 & 16 & 32 \\
\hline CST & $0.5-8$ & 101 & $\leq 0.5$ & $>8$ \\
\hline TGC & $0.25-4$ & 101 & 0.5 & 1 \\
\hline \multicolumn{5}{|l|}{ Enterobacter sp. } \\
\hline CFDC & $0.03-64$ & 14 & 0.5 & 1 \\
\hline $\mathrm{CT}$ & $0.03-64$ & 14 & 64 & $>64$ \\
\hline CAZ & $0.03-64$ & 14 & $>64$ & $>64$ \\
\hline CZA & $0.03-64$ & 14 & 1 & 2 \\
\hline FEP & $0.05-16$ & 14 & $>16$ & $>16$ \\
\hline MEM & $0.03-64$ & 14 & 8 & 16 \\
\hline CIP & $0.25-4$ & 14 & $>4$ & $>4$ \\
\hline ATM & $0.05-32$ & 14 & $>32$ & $>32$ \\
\hline AMK & $4-64$ & 14 & $\leq 4$ & $\leq 4$ \\
\hline $\mathrm{CST}$ & $0.5-8$ & 14 & $\leq 0.5$ & 8 \\
\hline TGC & $0.25-4$ & 14 & 0.5 & 1 \\
\hline
\end{tabular}

${ }^{\mathrm{a}} \mathrm{MIC}$, minimum inhibitory concentration; $\mathrm{MIC}_{50 / 90}$, MIC that inhibits $50 \%$ and $90 \%$ of the isolates, respectively 
Table 4 In vitro activities of cefiderocol (CFDC), ceftolozanetazobactam (CT), ceftazidime (CAZ), ceftazidime-avibactam (CZA), cefepime (FEP) meropenem (MEM), ciprofloxacin (CIP), aztreonam (ATM), amikacin (AMK), colistin (CST), and tigecycline (TGC) against Enterobacteriaceae

(E. coli, K. pneumoniae, and Enterobacter sp.) producing an OXA-48-type carbapenemase

\begin{tabular}{|c|c|c|c|c|}
\hline Organism group/antimicrobial agent & MIC range $(\mathrm{mg} / \mathrm{L})$ & No. & $\mathrm{MIC}_{50}{ }^{\mathrm{a}}$ & $\mathrm{MIC}_{90}{ }^{\mathrm{a}}$ \\
\hline \multicolumn{5}{|l|}{ Total Enterobacteriaceae } \\
\hline CFDC & $0.03-64$ & 154 & 0.25 & 2 \\
\hline $\mathrm{CT}$ & $0.03-64$ & 154 & 32 & $>64$ \\
\hline CAZ & $0.03-64$ & 154 & 64 & $>64$ \\
\hline CZA & $0.03-64$ & 154 & 0.5 & 4 \\
\hline FEP & $0.05-16$ & 154 & $>16$ & $>16$ \\
\hline MEM & $0.03-64$ & 154 & 1 & 32 \\
\hline CIP & $0.25-4$ & 154 & $>4$ & $>4$ \\
\hline ATM & $0.05-32$ & 154 & $>32$ & $>32$ \\
\hline AMK & $4-64$ & 154 & $\leq 4$ & 16 \\
\hline CST & $0.5-8$ & 154 & $\leq 0.5$ & 1 \\
\hline TGC & $0.25-4$ & 154 & $\leq 0.25$ & 1 \\
\hline \multicolumn{5}{|l|}{ Escherichia coli } \\
\hline CFDC & $0.03-64$ & 42 & 0.06 & 0.5 \\
\hline $\mathrm{CT}$ & $0.03-64$ & 42 & 2 & 32 \\
\hline $\mathrm{CAZ}$ & $0.03-64$ & 42 & 4 & 64 \\
\hline $\mathrm{CZA}$ & $0.03-64$ & 42 & 0.25 & 0.5 \\
\hline FEP & $0.05-16$ & 42 & 8 & $>16$ \\
\hline MEM & $0.03-64$ & 42 & 1 & 4 \\
\hline CIP & $0.25-4$ & 42 & $\leq 0.25$ & $>4$ \\
\hline ATM & $0.05-32$ & 42 & 32 & $>32$ \\
\hline AMK & $4-64$ & 42 & $\leq 4$ & $\leq 4$ \\
\hline $\mathrm{CST}$ & $0.5-8$ & 42 & $\leq 0.5$ & 1 \\
\hline TGC & $0.25-4$ & 42 & $\leq 0.25$ & $\leq 0.25$ \\
\hline \multicolumn{5}{|l|}{ Klebsiella pneumoniae } \\
\hline CFDC & $0.03-64$ & 88 & 0.25 & 1 \\
\hline $\mathrm{CT}$ & $0.03-64$ & 88 & 64 & $>64$ \\
\hline CAZ & $0.03-64$ & 88 & 64 & $>64$ \\
\hline CZA & $0.03-64$ & 88 & 0.5 & $>64$ \\
\hline FEP & $0.05-16$ & 88 & $>16$ & $>16$ \\
\hline MEM & $0.03-64$ & 88 & 1 & 64 \\
\hline CIP & $0.25-4$ & 88 & $>4$ & $>4$ \\
\hline ATM & $0.05-32$ & 88 & $>32$ & $>32$ \\
\hline AMK & $4-64$ & 88 & $\leq 4$ & $>64$ \\
\hline $\mathrm{CST}$ & $0.5-8$ & 88 & $\leq 0.5$ & 1 \\
\hline $\mathrm{TGC}$ & $0.25-4$ & 88 & 0.5 & 1 \\
\hline \multicolumn{5}{|l|}{ Enterobacter sp. } \\
\hline CFDC & $0.03-64$ & 24 & 1 & 4 \\
\hline $\mathrm{CT}$ & $0.03-64$ & 24 & 32 & $>64$ \\
\hline CAZ & $0.03-64$ & 24 & $>64$ & $>64$ \\
\hline CZA & $0.03-64$ & 24 & 0.5 & 2 \\
\hline FEP & $0.05-16$ & 24 & $>16$ & $>16$ \\
\hline MEM & $0.03-64$ & 24 & 1 & 8 \\
\hline CIP & $0.25-4$ & 24 & $>4$ & $>4$ \\
\hline ATM & $0.05-32$ & 24 & $>32$ & $>32$ \\
\hline AMK & $4-64$ & 24 & 8 & 8 \\
\hline $\mathrm{CST}$ & $0.5-8$ & 24 & $\leq 0.5$ & 1 \\
\hline TGC & $0.25-4$ & 24 & 1 & 1 \\
\hline
\end{tabular}

${ }^{\mathrm{a}} \mathrm{MIC}$, minimum inhibitory concentration; $\mathrm{MIC}_{50 / 90}$, MIC that inhibits $50 \%$ and $90 \%$ of the isolates, respectively 
Table 5 In vitro activities of cefiderocol (CFDC), ceftolozanetazobactam (CT), ceftazidime (CAZ), ceftazidime-avibactam (CZA), cefepime (FEP), meropenem (MEM) ciprofloxacin (CIP), aztreonam (ATM), amikacin (AMK), colistin (CST), and tigecycline (TGC) against Enterobacteriaceae

(E. coli, K. pneumoniae, and Enterobacter sp.) producing NDM, VIM, or IMP carbapenemases

\begin{tabular}{|c|c|c|c|c|}
\hline Organism group/antimicrobial agent & MIC range $(\mathrm{mg} / \mathrm{L})$ & No. & $\mathrm{MIC}_{50}{ }^{\mathrm{a}}$ & $\mathrm{MIC}_{90}{ }^{\mathrm{a}}$ \\
\hline \multicolumn{5}{|l|}{ Total Enterobacteriaceae } \\
\hline CFDC & $0.03-64$ & 134 & 1 & 4 \\
\hline $\mathrm{CT}$ & $0.03-64$ & 134 & $>64$ & $>64$ \\
\hline CAZ & $0.03-64$ & 134 & $>64$ & $>64$ \\
\hline CZA & $0.03-64$ & 134 & $>64$ & $>64$ \\
\hline FEP & $0.05-16$ & 134 & $>16$ & $>16$ \\
\hline MEM & $0.03-64$ & 134 & 32 & $>64$ \\
\hline CIP & $0.25-4$ & 134 & $>4$ & $>4$ \\
\hline ATM & $0.05-32$ & 134 & $>32$ & $>32$ \\
\hline AMK & $4-64$ & 134 & 8 & $>64$ \\
\hline CST & $0.5-8$ & 134 & $\leq 0.5$ & 1 \\
\hline TGC & $0.25-4$ & 134 & $\leq 0.25$ & 1 \\
\hline \multicolumn{5}{|l|}{ Escherichia coli } \\
\hline CFDC & $0.03-64$ & 67 & 1 & 16 \\
\hline $\mathrm{CT}$ & $0.03-64$ & 67 & $>64$ & $>64$ \\
\hline CAZ & $0.03-64$ & 67 & $>64$ & $>64$ \\
\hline $\mathrm{CZA}$ & $0.03-64$ & 67 & $>64$ & $>64$ \\
\hline FEP & $0.05-16$ & 67 & $>16$ & $>16$ \\
\hline MEM & $0.03-64$ & 67 & 64 & $>64$ \\
\hline CIP & $0.25-4$ & 67 & $>4$ & $>4$ \\
\hline ATM & $0.05-32$ & 67 & $>32$ & $>32$ \\
\hline AMK & $4-64$ & 67 & 8 & $>64$ \\
\hline $\mathrm{CST}$ & $0.5-8$ & 67 & $\leq 0.5$ & 1 \\
\hline TGC & $0.25-4$ & 67 & $\leq 0.25$ & $\leq 0.25$ \\
\hline \multicolumn{5}{|l|}{ Klebsiella pneumoniae } \\
\hline CFDC & $0.03-64$ & 38 & 1 & 4 \\
\hline $\mathrm{CT}$ & $0.03-64$ & 38 & $>64$ & $>64$ \\
\hline CAZ & $0.03-64$ & 38 & $>64$ & $>64$ \\
\hline CZA & $0.03-64$ & 38 & $>64$ & $>64$ \\
\hline FEP & $0.05-16$ & 38 & $>16$ & $>16$ \\
\hline MEM & $0.03-64$ & 38 & 32 & $>64$ \\
\hline CIP & $0.25-4$ & 38 & $>4$ & $>4$ \\
\hline ATM & $0.05-32$ & 38 & $>32$ & $>32$ \\
\hline AMK & $4-64$ & 38 & 16 & $>64$ \\
\hline CST & $0.5-8$ & 38 & $\leq 0.5$ & 1 \\
\hline TGC & $0.25-4$ & 38 & 1 & 2 \\
\hline \multicolumn{5}{|l|}{ Enterobacter sp. } \\
\hline CFDC & $0.03-64$ & 29 & 1 & 4 \\
\hline $\mathrm{CT}$ & $0.03-64$ & 29 & $>64$ & $>64$ \\
\hline CAZ & $0.03-64$ & 29 & $>64$ & $>64$ \\
\hline CZA & $0.03-64$ & 29 & $>64$ & $>64$ \\
\hline FEP & $0.05-16$ & 29 & $>16$ & $>16$ \\
\hline MEM & $0.03-64$ & 29 & 16 & 64 \\
\hline CIP & $0.25-4$ & 29 & 1 & $>4$ \\
\hline ATM & $0.05-32$ & 29 & 16 & $>32$ \\
\hline AMK & $4-64$ & 29 & $\leq 4$ & $>64$ \\
\hline $\mathrm{CST}$ & $0.5-8$ & 29 & $\leq 0.5$ & $\leq 0.5$ \\
\hline TGC & $0.25-4$ & 29 & 0.5 & 1 \\
\hline
\end{tabular}

${ }^{\mathrm{a}} \mathrm{MIC}$, minimum inhibitory concentration; $\mathrm{MIC}_{50 / 90}$, MIC that inhibits $50 \%$ and $90 \%$ of the isolates, respectively 
Table 6 In vitro activities of cefiderocol (CFDC), ceftolozanetazobactam (CT), meropenem (MEM), ceftazidime (CAZ), ceftazidime-avibactam (CZA), colistin (CST), aztreonam (ATM), amikacin (AMK), ciprofloxacin (CIP), cefepime (FEP), and tigecycline (TGC) against $P$. aeruginosa producing a carbapenemase (either IMP, KPC, VIM, SPM, or GIM)

\begin{tabular}{|c|c|c|c|c|}
\hline Organism group/antimicrobial agent & MIC range $(\mathrm{mg} / \mathrm{L})$ & No. & $\mathrm{MIC}_{50}{ }^{\mathrm{a}}$ & $\mathrm{MIC}_{90}{ }^{\mathrm{a}}$ \\
\hline \multicolumn{5}{|l|}{ Pseudomonas aeruginosa } \\
\hline CFDC & $0.03-64$ & 30 & 0.5 & 2 \\
\hline $\mathrm{CT}$ & $0.03-64$ & 30 & $>64$ & $>64$ \\
\hline CAZ & $0.03-64$ & 30 & $>64$ & $>64$ \\
\hline $\mathrm{CZA}$ & $0.03-64$ & 30 & $>64$ & $>64$ \\
\hline FEP & $0.05-16$ & 30 & $>16$ & $>16$ \\
\hline MEM & $0.03-64$ & 30 & 64 & $>64$ \\
\hline CIP & $0.25-4$ & 30 & $>4$ & $>4$ \\
\hline ATM & $0.05-32$ & 30 & 8 & 64 \\
\hline AMK & $4-64$ & 30 & 8 & $>64$ \\
\hline CST & $0.5-8$ & 30 & $\leq 0.5$ & 1 \\
\hline TGC & $0.25-4$ & 30 & 8 & $>4$ \\
\hline
\end{tabular}

${ }^{\mathrm{a}} \mathrm{MIC}$, minimum inhibitory concentration; $\mathrm{MIC}_{50 / 90}$, MIC that inhibits $50 \%$ and $90 \%$ of the isolates, respectively $\geq 8)$, and tigecycline $(\leq 0.5 / \leq 1)$. Elevated $\mathrm{MIC}_{90}$ values for colistin were noted for 17 out of 101 strains for K. pneumoniae and 2 out of 14 strains for Enterobacter sp. For all other antibiotics, the $\mathrm{MIC}_{50 / 90}$ were superior or equal to the upper limit of the concentration range.

OXA-48-like producing Enterobacteriaceae were susceptible to a larger number of antibiotics compared to the KPC producers (Table 4). Again, cefiderocol is one of the antibiotics with the lowest $\mathrm{MIC}_{50 / 90}$ values, at 0.25 and $2 \mu \mathrm{g} / \mathrm{mL}$, respectively. Direct competitors were ceftazidime-avibactam $(0.5 / 4)$, meropenem $(1 / 32)$, amikacin $(\leq 4 / 16)$, colistin $(\leq 0.5 /$ $1)$, and tigecycline $(\leq 0.5 / \leq 1)$. Again, for Klebsiella sp., the $\mathrm{MIC}_{90}$ of CZA and MEM were affected by outliers and the $\mathrm{MIC}_{50}$ values should be considered instead.

For the enterobacterial isolates producing NDM, VIM, or IMP carbapenemases (Table 5), the only antibiotics that had strong activity $\left(\mathrm{MIC}_{50 / 90}\right)$ were cefiderocol (1/4), colistin $(\leq 0.5 / \leq 1)$, and tigecycline $(\leq 0.25 / \leq 1)$.
Colistin-resistant strains, mainly Enterobacteriaceae, had high susceptibility to cefiderocol $(\leq 0.5 / \leq 2)$ and some activity for ceftolozane-tazobactam in the case of E. coli $(0.25 />64)$, ceftazidime-avibactam $(0.5 />64)$, meropenem $(0.12 / 64)$, amikacin $(\leq 4 / 16)$, and tigecycline $(\leq 1 / \leq 1)$. Except for cefiderocol and tigecycline, the $\mathrm{MIC}_{90}$ values were close to or above the upper limit of the concentration range of the tested antibiotics for the Enterobacteriaceae being resistant to colistin. This could be explained by additional resistance traits, such as expression of genes encoding carbapenemases and ESBLs, in particular for K. pneumoniae and Enterobacter sp. The level of resistance to colistin $\left(\mathrm{MIC}_{90}=2\right)$ and of meropenem $\left(\mathrm{MIC}_{90}=1\right)$ was lower for $E$. coli than that noted for K. pneumoniae and Enterobacter sp.

Carbapenemase-producing $P$. aeruginosa were susceptible only to cefiderocol $(0.5 / 2)$ and colistin $(\leq 0.5 / 1)$ (Table 6$)$. The same resistance trend was observed for carbapenemaseproducing A. baumannii strains [CFDC (0.12/4) and CST
Table 7 In vitro activities of cefiderocol (CFDC), ceftolozanetazobactam (CT), meropenem (MEM), ceftazidime (CAZ) ceftazidime-avibactam (CZA), colistin (CST), aztreonam (ATM), amikacin (AMK), ciprofloxacin (CIP), cefepime (FEP), and tigecycline (TGC) against A. baumannii producing an OXAtype carbapenemase (either OXA$23,-40,-58$, or -72 )

\begin{tabular}{lcccc}
\hline Organism group/antimicrobial agent & MIC range (mg/L) & No. & $\mathrm{MIC}_{50}{ }^{\mathrm{a}}$ & $\mathrm{MIC}_{90}{ }^{\mathrm{a}}$ \\
\hline $\begin{array}{l}\text { Acinetobacter baumannii } \\
\text { CFDC }\end{array}$ & $0.03-64$ & & & \\
CT & $0.03-64$ & 85 & 0.12 & 4 \\
CAZ & $0.03-64$ & 85 & 16 & $>64$ \\
CZA & $0.03-64$ & 85 & $>64$ & $>64$ \\
FEP & $0.05-16$ & 85 & 16 & 64 \\
MEM & $0.03-64$ & 85 & 32 & $>16$ \\
CIP & $0.25-4$ & 85 & $>4$ & 64 \\
ATM & $0.05-32$ & 85 & $>32$ & $>4$ \\
AMK & $4-64$ & 85 & 64 & $>64$ \\
CST & $0.5-8$ & 85 & 0.5 & 1 \\
TGC & $0.25-4$ & 85 & 1 & 2 \\
\hline
\end{tabular}

${ }^{\text {a }} \mathrm{MIC}$, minimum inhibitory concentration; $\mathrm{MIC}_{50 / 90}$, MIC that inhibits $50 \%$ and $90 \%$ of the isolates, respectively 
$(0.5 / 1)]$, except that they were also susceptible to tigecycline $(1 / 2)$ (Table 7). The only unexpected result is the overall low activity of ceftolozane-tazobactam against those $P$. aeruginosa isolates.

Among the 753 isolates tested, only 24 isolates exhibited an MIC value of cefiderocol $\geq 8 \mu \mathrm{g} / \mathrm{mL}$, among which $45 \%$ were NDM producers $(n=11), 30 \%$ were OXA-23-producing A. baumannii $(n=7)$, and two VEB-, one SHV-, one VIM-, and one OXA-48-like producers among $K$. pneumoniae, $P$. aeruginosa, and Enterobacter sp. Noteworthy, cefiderocol was active against 68 out of 79 NDM producers, while most of the NDM producers co-produced other $\beta$-lactam resistance mechanisms, such as ESBLs (mostly CTX-M-15), porin defect, plasmid-mediated cephalosporinases, and other carbapenemases (data not shown). Cefiderocol was more active ( $\mathrm{MIC}_{90} 2-4 \mathrm{mg} / \mathrm{L}$ ) than the comparators ( $\mathrm{MIC}_{90}>4$ to $>64 \mathrm{mg} /$ L) (cephalosporins, carbapenem, fluoroquinolone, and monobactam) against all the tested strains. The only comparators with equal activity were colistin and tigecycline, with the limitation that tigecycline was not active against $P$. aeruginosa. Finally, it should be emphasized that cefiderocol displays much favorable pharmacokinetic parameters (tissue diffusion and use in renal impairment) than colistin and tigecycline [19], which will be an important factor for choosing adequate therapy of infections due to multidrug infections.

\section{Compliance with ethical standards}

Funding This work was funded by grants from the University of Fribourg and from Shionogi \& Co., Ltd.

Conflict of interest None to declare.

Ethical approval Not applicable.

Informed consent Not applicable.

\section{References}

1. Pitout JDD, Laupland KB (2008) Extended-spectrum $\beta$-lactamaseproducing Enterobacteriaceae: an emerging public-health concern. Lancet Infect Dis 8(3):159-166

2. Knothe H, Shah P, Krcmery V, Antal M, Mitsuhashi S (1983) Transferable resistance to cefotaxime, cefoxitin, cefamandole and cefuroxime in clinical isolates of Klebsiella pneumoniae and Serratia marcescens. Infection 11(6):315-317

3. Philippon A, Labia R, Jacoby G (1989) Extended-spectrum $\beta$ lactamases. Antimicrob Agents Chemother 33(8):1131-1136

4. Livermore DM (2003) Bacterial resistance: origins, epidemiology, and impact. Clin Infect Dis 36:S11-S23

5. Gupta N, Limbago BM, Patel JB, Kallen AJ (2011) Carbapenemresistant Enterobacteriaceae: epidemiology and prevention. Clin Infect Dis 53(1):60-67

6. Kanj SS, Kanafani ZA (2011) Current concepts in antimicrobial therapy against resistant gram-negative organisms: extended- spectrum $\beta$-lactamase-producing Enterobacteriaceae, carbapenemresistant Enterobacteriaceae, and multidrug-resistant Pseudomonas aeruginosa. Mayo Clin Proc 86(3):250-259

7. Poirel L, Kieffer N, Liassine N, Thanh D, Nordmann P (2016) Plasmid-mediated carbapenem and colistin resistance in a clinical isolate of Escherichia coli. Lancet Infect Dis 16(3):281

8. Liu YY, Wang Y, Walsh TR, Yi LX, Zhang R, Spencer J, Doi Y, Tian G, Dong B, Huang X, Yu LF, Gu D, Ren H, Chen X, Lv L, He D, Zhou H, Liang Z, Liu JH, Shen J (2016) Emergence of plasmidmediated colistin resistance mechanism MCR-1 in animals and human beings in China: a microbiological and molecular biological study. Lancet Infect Dis 16(2):161-168

9. Haenni M, Poirel L, Kieffer N, Châtre P, Saras E, Métayer V, Dumoulin R, Nordmann P, Madec JY (2016) Co-occurrence of extended spectrum $\beta$ lactamase and MCR-1 encoding genes on plasmids. Lancet Infect Dis 16(3):281-282

10. Hasman H, Hammerum AM, Hansen F, Hendriksen RS, Olesen B, Agersø Y, Zankari E, Leekitcharoenphon P, Stegger M, Kaas RS, Cavaco LM, Hansen DS, Aarestrup FM, Skov RL (2015) Detection of $m c r-1$ encoding plasmid-mediated colistin-resistant Escherichia coli isolates from human bloodstream infection and imported chicken meat, Denmark 2015. Euro Surveill 20(49)

11. Kohira N, West J, Ito A, Ito-Horiyama T, Nakamura R, Sato T, Rittenhouse S, Tsuji M, Yamano Y (2015) In vitro antimicrobial activity of a siderophore cephalosporin, S-649266, against Enterobacteriaceae clinical isolates, including carbapenemresistant strains. Antimicrob Agents Chemother 60(2):729-734

12. Ito A, Nishikawa T, Matsumoto S, Yoshizawa H, Sato T, Nakamura R, Tsuji M, Yamano Y (2016) Siderophore cephalosporin cefiderocol utilizes ferric iron transporter systems for antibacterial activity against Pseudomonas aeruginosa. Antimicrob Agents Chemother 60(12):7396-7401

13. Tillotson GS (2016) Trojan horse antibiotics - a novel way to circumvent gram-negative bacterial resistance? Infect Dis (Auckl) 9: $45-52$

14. Ito-Horiyama T, Ishii $\mathrm{Y}$, Ito A, Sato T, Nakamura R, Fukuhara N, Tsuji M, Yamano Y, Yamaguchi K, Tateda K (2016) Stability of novel siderophore cephalosporin S-649266 against clinically relevant carbapenemases. Antimicrob Agents Chemother 60(7):43844386

15. Ito A, Kohira N, Bouchillon SK, West J, Rittenhouse S, Sader HS, Rhomberg PR, Jones RN, Yoshizawa H, Nakamura R, Tsuji M, Yamano Y (2016) In vitro antimicrobial activity of S-649266, a catechol-substituted siderophore cephalosporin, when tested against non-fermenting gram-negative bacteria. J Antimicrob Chemother 71(3):670-677

16. Clinical and Laboratory Standards Institute (CLSI) (2015) Methods for dilution antimicrobial susceptibility tests for bacteria that grow aerobically; Approved standards - tenth edition. CLSI document M07-A10. CLSI, Wayne, PA

17. Huband MD, Ito A, Tsuji M, Sader HS, Fedler KA, Flamm RK (2017) Cefiderocol MIC quality control ranges in iron-depleted cation-adjusted Mueller-Hinton broth using a CLSI M23-A4 multi-laboratory study design. Diagn Microbiol Infect Dis 88(2): 198-200

18. Clinical and Laboratory Standards Institute (CLSI) (2015) Performance standards for antimicrobial susceptibility testing; Twenty-fifth informational supplement. CLSI document M100S25. CLSI, Wayne, PA

19. Katsube T, Wajima T, Ishibashi T, Camilo Arjona Ferreira J, Echols R (2017) Pharmacokinetic/pharmacodynamic modeling and simulation of cefiderocol, a parenteral siderophore cephalosporin, for dose adjustment based on renal function. Antimicrob Agents Chemother 61(1). pii: e01381-16 